\title{
Agreement between Patient-Generated Subjective Global Assessment Short Form and Short Nutritional Assessment Questionnaire in a selected clinical population
}

\author{
Priya Dewansingh ${ }^{1}$, Margreet Euwes ${ }^{2}$, Wim P. Krijnen ${ }^{1,3,4}$, Jaap H. Strijbos ${ }^{5}$, Faith D. \\ Ottery ${ }^{1,6}$, Cees P. van der Schans ${ }^{1,7,8}$, Harriët Jager-Wittenaar ${ }^{1,9}$ \\ 1 Research Group Healthy Ageing, Allied Health Care and Nursing, Hanze University of Applied Sciences, Groningen, The Netherlands \\ 2 Acute Care Rehabilitation, Nij Smellinghe Hospital, Drachten, The Netherlands \\ 4 Faculty of Mathematics and Natural Sciences, University of Groningen, Groningen, The Netherlands \\ 4 Faculty of Medical Sciences, University Medical Center Groningen, Groningen, The Netherlands \\ 6 Ottery \& Associates, Vernon Hills (Chicago), United States \\ 7 Department of Rehabilitation Medicine, University of Groningen, University Medical Center Groningen, Groningen, The Netherl ands \\ 8 Department of Health Psychology Research, University of Groningen, University Medical Center Groningen, Groningen, The Neth erlands \\ 9 Department of Maxillofacial Surgery, University of Groningen, University Medical Center Groningen, Groningen, The Netherlan ds
}

\section{Aim}

We aimed to assess agreement between the

Patient-Generated Subjective Global Assessment Short

Form (PG-SGA SF) and the Short Nutritional

Assessment Questionnaire (SNAQ), and their agreement with the full PG-SGA, in a selected clinical population.

\section{Conclusion}

Agreement between PG-SGA SF and SNAQ is poor. Agreement between SNAQ and full PG-SGA (reference) is also poor. PG-SGA SF categorizes 2.6 times more patients at risk of malnutrition than SNAQ, which facilitates triaging for proactive and interdisciplinary interventions to prevent or treat malnutrition.

\section{Background}

$\mathrm{n}$ the Dutch hospital Nij Smellinghe, the SNAQ is used to screen for risk of malnutrition. Since 2014, the PG-SGA including the PG-SGA Short Form ${ }^{1}$, an interdisciplinary malnutrition tool that can be used to screen, monitor and assess malnutrition and its risk factors, is available in Dutch. ${ }^{2}$

\begin{tabular}{|c|c|c|c|c|c|}
\hline & $\begin{array}{l}\text { Risk of } \\
\text { malnutrition SNAQ }\end{array}$ & $\begin{array}{l}\text { Risk of malnutrition } \\
\text { PG-SGA SF }\end{array}$ & $\begin{array}{l}\text { Agreement } \\
\text { PG-SGA SF and SNAQ }\end{array}$ & $\begin{array}{l}\text { Agreement } \\
\text { SNAQ and PG-SGA }\end{array}$ & $\begin{array}{l}\text { Agreement } \\
\text { PG-SGA SF and PG-SGA }\end{array}$ \\
\hline Lung disease $(n=130)$ & $24 \%$ & $72 \%$ & $0.19 ; 95 \% \mathrm{Cl} 0.09-0.29$ & $0.24 ; 95 \% \mathrm{Cl} 0.10-0.35$ & $0.64 ; 95 \%$ Cl 0.55-0.74 \\
\hline Cardiology $(n=101)$ & $17 \%$ & $49 \%$ & $0.37 ; 95 \% \mathrm{Cl} 0.23-0.52$ & $0.37 ; 95 \%$ Cl 0.21-0.53 & $0.66 ; 95 \% \mathrm{Cl} 0.55-0.78$ \\
\hline Surgery $(n=224)$ & $26 \%$ & $56 \%$ & $0.31 ; 95 \% \mathrm{Cl} 0.21-0.41$ & $0.36 ; 95 \% \mathrm{Cl} 0.25-0.47$ & $0.69 ; 95 \% \mathrm{Cl} 0.62-0.75$ \\
\hline Colon/rectum carcinoma $(n=21)$ & $19 \%$ & $38 \%$ & $0.48 ; 95 \% \mathrm{Cl} 0.12-0.84$ & $0.65 ; 95 \% \mathrm{Cl} 0.33-0.98$ & $0.77 ; 95 \% \mathrm{Cl} 0.50-0.10$ \\
\hline COPD outpatient $(n=19 ; 19 ; 20)$ & $19 \%$ & $38 \%$ & $0.48 ; 95 \% \mathrm{Cl} 0.03-0.93$ & $0.38 ; 95 \% \mathrm{Cl}-0.20-0.96$ & $0.48 ; 95 \% \mathrm{Cl} 0.03-0.93$ \\
\hline Orthopedic $(n=35 ; 35 ; 37)$ & $0 \%$ & $30 \%$ & * & * & $0.35 ; 95 \% \mathrm{Cl} 0.04-0.65$ \\
\hline Total $(n=530 ; 530 ; 533)$ & $22 \%$ & $55 \%$ & $0.30 ; 95 \% \mathrm{Cl} 0.24-0.36$ & $0.34 ; 95 \% \mathrm{Cl} 0.27-0.42$ & $0.68 ; 95 \% \mathrm{Cl} 0.63-0.72$ \\
\hline
\end{tabular}

Table 1. Agreement between the Short nutritional Assessment Questionnaire (SNAQ), Patient-Generated Subjective Global Assessment Short Form (PG-SGA SF), and PG-SGA

Not calculated (all patients scored low risk with the SNAQ)

Results

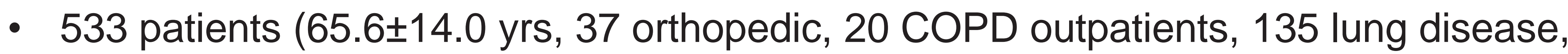
103 cardiologic, 21 colon/rectum cancer, and 217 surgery) were included

- $50 \%$ of the patients who were screened as low risk by the SNAQ were screened as medium/high risk by the PG-SGA SF.

- Agreement between PG-SGA SF and SNAQ was $\mathrm{k}=0.30 ; 95 \% \mathrm{Cl}$ 0.24-0.36.

- Agreement between PG-SGA SF and SNAQ per patient group is shown in Table 1.
- Pre-surgery orthopedic patients, COPD outpatients, lung disease inpatients, cardiologic inpatients, colon/rectum cancer outpatients, and surgery inpatients from a regional hospital in The Netherlands participated in the study.

\section{SGA SF, and malnutrition by full PG-SGA.}

The SNAQ includes four (yes/no) questions about weight loss, appetite, and the use of nutritional supplements and/or tube feeding.

G-SGA SF includes four boxes: Weight, Food intake

Risk of malnutrition was categorized as: Iow (PG-SGA SF 0-3, SNAQ 0-1 points), medium (resp. 4-8 and 2 points), and high risk (resp. $\geq 9$ and $\geq 3$ points). Malnutrition was defined as $P G-S G A$ malnourished).

- Weighted kappa ( $\mathrm{K}$ ) was used to analyze agreement between the PG-SGA SF and SNAQ, and agreement between respectively the PG-SGA SF and SNAQ and the full PG-SGA (reference).

\section{share your talent. move the world.}

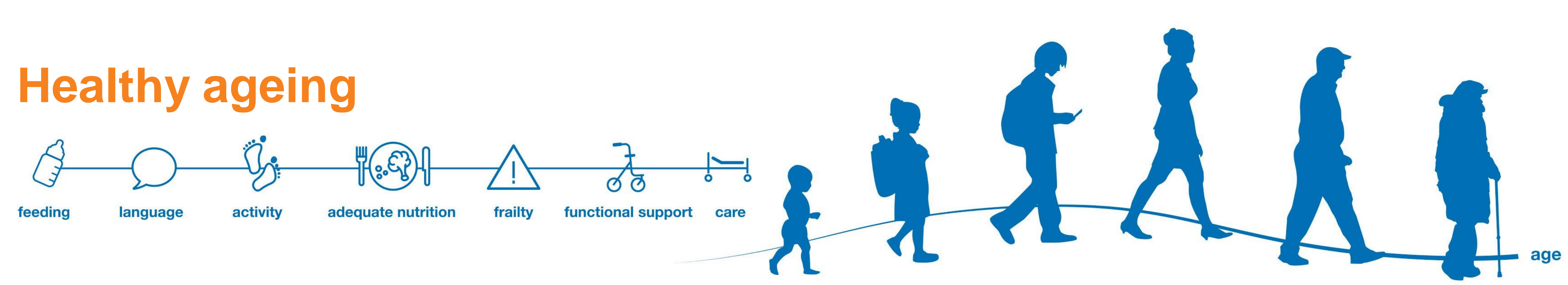

\section{References}

1. Ottery FD. Definition of standardized nutritional assessment and interventional pathways in oncology. Nutrition 1996;12(1 Suppl): S15-9.

2. Sealy MJ, Haß U, Ottery FD, Roodenburg JLN, Van der Schans CP, Jager-Wittenaar $\mathrm{H}$. Translation and cultural adaptation of the Scored Patient-Generated Subjective Global Assessment (PG-SGA): an interdisciplinary nutritional instrument appropriate for Dutch cancer patients. Cancer Nursing May 17 [Epub ahead of print]

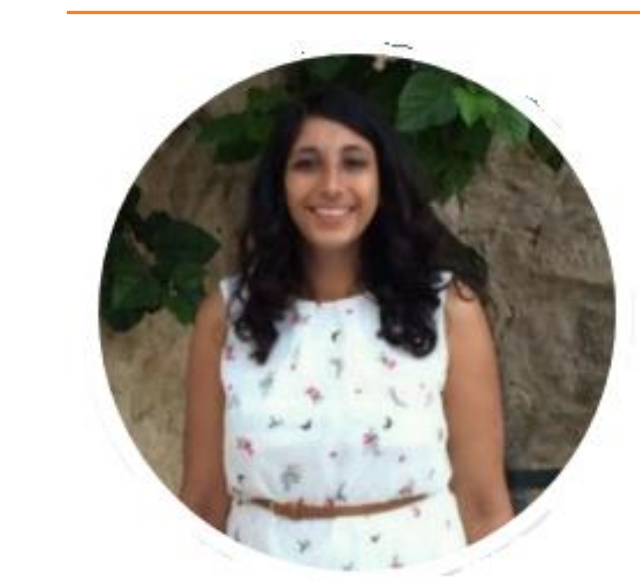

Contact detaills Priya Dewansingh p.dewansingh@pl.hanze.nl 